\title{
Spitzer Observations of Tidal Dwarf Galaxies
}

\author{
Sarah J. U. Higdon ${ }^{1,2}$ and James L. Higdon ${ }^{1}$ \\ ${ }^{1}$ Physics Department, Georgia Southern University, Statesboro, GA 30460, \\ ${ }^{2}$ Astronomy Department, Cornell University, Ithaca, NY 14853 \\ email: shigdon@georgiasouthern.edu
}

\begin{abstract}
We present Spitzer observations of Tidal Dwarf Galaxies (TDGs) in three interacting systems: NGC 5291, Arp 105 \& Stephan's Quintet. The spectra show bright emission from polyaromatic hydrocarbons (PAHs), nebular lines and warm molecular hydrogen, characteristic of recent episodes of star formation. The PAH emission that falls in the IRAC $8.0 \mu \mathrm{m}$ band leads to the TDGs having an extremely red IRAC color, with [4.5] $-[8.0]>3$. The emission from PAHs is characterized by a model with mainly neutral 100-C PAH atoms.
\end{abstract}

Keywords. galaxies: dwarf, galaxies: evolution, galaxies: formation, infrared: galaxies

\section{Introduction}

Mergers of gas rich dusty spirals can form $>100 \mathrm{kpc}$ tidal tails (Toomre \& Toomre 1972). Tidal Dwarf Galaxies (TDGs) condense out of these tails, and may make up $1 / 3$ of the dwarf population in compact groups (Hunsberger et al. 1996). Here we investigate the emission from Polycyclic Aromatic Hydrocarbons (PAHs) in three TDG systems:

NGC 5291/Seashell (western edge of Abell 3574). Disturbed lenticular. Large fragmented HI ring in a mainly 8'eastern arc. Two TDGs NGC 5291N \& NGC 5291S \& 13 TDG candidates (Spitzer observations: Higdon, Higdon \& Marshall 2006).

Arp105/The Guitar (Abell 1185). NGC 3561A(spiral) \& NGC 3561B(lenticular). Ambartzumian knot (TDG at tip of southern tail) \& 5 TDG candidates.

Stephans Quintet (Arp 319) Prototype compact group (Hickson 92). NGC 7317(E), NGC 7318A(E), NGC 7318B (Sbc pec), NGC 7319 (Sbc pec Sey 2) and NGC 7320 (Sd foreground galaxy). Two $100 \mathrm{kpc}$ parallel tails from NGC 7319 towards NGC 7320c. $40 \mathrm{kpc}$ shock front in intra-group medium. TDGs: SQ-A, SQ-B (Arp 1973), 13 TDG candidates (Hunsberger et al. 1996).

\section{TDGs Are PAH Rich \& Have Extreme IRAC Colors}

Spitzer Infrared Array Camera (IRAC) false color images showing the PAH-rich TDGs are available at our web site $\dagger$. The strong PAH emission is used to identify the TDGs in the IRAC two-color diagram shown in Figure 1. The horizontal and vertical lines mark the $[4.5]-[8.0]$ and [3.6] - [4.5] color zero points. Galaxies are sorted into 3 quadrants: Elliptical/Spheroidal (bottom left). AGN/rising SED (top right). Star formation (bottom right). Compared to spiral galaxies and Blue Compact Galaxies (BCDs) the TDGs occupy the extreme right with [4.5] - [8.0] > 3 (Higdon, Higdon \& Marshall 2006). The knot in the ring galaxy, AM0644-74 is consistent with the TDG color (Higdon \& Higdon 2007).

$\dagger$ http://cheller.phy.georgiasouthern.edu/ shigdon/tdg/tdg.html 


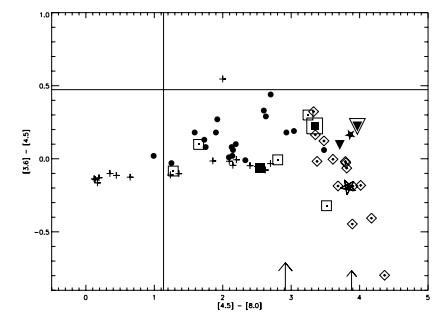

Figure 1. IRAC Two-Color Diagram: NGC 5291: NGC 5291N (solid star), NGC 5291S (framed star), TDG candidates (framed diamonds). SQ-A \& SQ-B (triangles). Arp 105: TDG candidates (framed squares) \& Ambartzumian knot (framed large square). Star forming regions in the Cartwheel (large arrow) and in AM0644-74 (small arrow). For comparison: Hubble morphological sequence ( +, Pahre et al 2004); KISS BCD Sample (small filled circle, Rosenburg et al. 2005).

\section{TDGs Have Mainly Neutral $\sim 100-C$ atom PAHs \& Warm $\mathbf{H}_{2}$}

Spectra, which were obtained with the Spitzer low resolution Infrared Spectrograph and extracted using SMART (Higdon et al. 2004), are rich in PAHs, with $\mathrm{H}_{2}$ in NGC $5291 \mathrm{~N} \& \mathrm{~S}$ and NGC 7319A; and [SIV] in NGC 5291N \& Ambartzumian Knot. The relative intensities of the $11.3 / 7.7 \mu \mathrm{m}$ and $6.2 / 7.7 \mu \mathrm{m}$ PAH features were measured using PAHFIT (Smith \& Draine 2007) and compared to models in Figure 16 from Draine \& Li (2001). The PAHs in NGC 5291 N\&S are consistent with 100 C-atoms. They are similar to the average PAH size of 27 normal galaxies (Helou 2000), but with a slightly higher ion fraction. $\mathrm{PAH}$ clumps are $\sim 4 \mathrm{x}$ bigger in Ambartzumian knot, whilst those in NGC 7319A are smaller and mostly neutral.

\section{Conclusions}

TDGs and knots of star formation in tidal streams tend to have disturbed morphologies, with recent episodes of star formation, as evidenced by emission from PAHs (modeled as mainly neutral 100-C PAHs), nebular lines, and warm $\mathrm{H}_{2}$. They can be identified by their [4.5] - [8.0] $>3$ color. As members of the Arecibo Legacy Fast ALFA (ALFALFA) Undergraduate team, we will expand this study to include new tidal features discovered in the ALFALFA Survey. Any found without visible perturbers are potential candidates for small dark matter halo interactions.

\section{Acknowledgements}

Sarah thanks both the I.A.U. and the Physics Dept., Georgia Southern University for travel grants. The Spitzer Space Telescope is operated by the Jet Propulsion Laboratory, Caltech, under NASA contract 1407. Support for this work was provided by NASA, through contract 1257184, issued by JPL/Caltech. We used the most excellent NED.

\section{References}

Draine, B. T., \& Li, A. 2001, ApJ, 551, 807

Helou, G., Lu, N. Y., Werner, M. W., Malhotra, S., \& Silbermann, N. 2000 ApJ, 532, L21

Higdon, S. J. U., et al. 2004 PASP, 116, 975

Higdon, S. J. U., Higdon, J. L., \& Marshall, J. 2006, ApJ, 640, 768

Higdon, S. J. U., Higdon, J. L. 2007 in prep.

Hunsberger, S. D., Charlton, J., \& Zaritsky, D. 1996 ApJ, 462, 50

Pahre, M. A., Ashby, M. L., Fazio, G. G., \& Willner, S. P. 2004 ApJS, 154, 235

Smith, J. D. T., \& Draine, B. T., et al., 2007, ApJ,656, 770

Toomre, A. \& Toomre, J. 1972, ApJ, 178, 623 